\title{
Ring-Opening Metathesis Polymerization of Norbornene with Catecholato Complexes of Tungsten(VI) as Effective Catalyst Precursors
}

\author{
Yuushou NAKAYAMA, Kouhei KATSUDA, and Hajime YASUdA ${ }^{\dagger}$ \\ Department of Applied Chemistry, Graduate School of Engineering, Hiroshima University, \\ Higashi-Hiroshima 739-8527, Japan
}

(Received July 4, 2003; Accepted August 25, 2003)

\begin{abstract}
Catalysis of catecholatotungsten(VI) complexes for the ring-opening metathesis polymerization (ROMP) of norbornene (NBE) and dicyclopentadiene (DCPD) was studied. Bis(catecholato) complex, $\mathrm{WCl}_{2}(\mathrm{cat})_{2}(\mathbf{2}$, $\mathrm{cat}=$ catecholato), was found to be particularly active for the ROMP of norbornene in the presence of AlEt 3 as a cocatalyst to give polymers with relatively narrow molecular weight distributions. A bulky catecholato complex, $\mathrm{WCl}_{2}\left({ }^{\mathrm{t}} \mathrm{Bucat}_{2}(\mathbf{3}\right.$, ${ }^{\mathrm{t} B u c a t}=3,5$-di-tert-butylcatecholato), afforded poly(NBE) with high cis-content. The use of modified methylaluminoxane (MMAO, Type $3 \mathrm{~A}$ in toluene) as a cocatalyst improved activities at low temperature $\left(-78^{\circ} \mathrm{C}\right)$. A mono(catecholato) complex, $\mathrm{WCl}_{4}$ (cat) $\mathbf{1}$, showed high activity for the ROMP of a bulkier monomer, DCPD, to give poly(DCPD) with high cis-content.

KEY WORDS Ring-opening Metathesis Polymerization / Norbornene / Dicyclopentadiene / Tungsten / Catecholato / 2,6-Dimethylphenoxo /
\end{abstract}

Polynorbornene is an interesting polymer for its special properties such as shape memory resin and oil-absorbing rubber. Hydrogenated polynorbornene derivatives have high transparency and low glass transition point suitable for optical applications. Classical catalysts such as $\mathrm{WCl}_{6} / \mathrm{AlEt}_{3}$ have been used for ring-opening metathesis polymerization (ROMP) of norbornene (NBE). ${ }^{1}$ These classical catalyst systems are easily available and highly active, however, they have some disadvantages: high sensitivity to air and moisture, poor controllability of the molecular weight distribution and microstructure of the resulting polymers. Recent development of organometallic chemistry achieved controlled ROMP catalyzed by well-defined alkylidene complexes such as $\mathrm{W}(=$ $\mathrm{NAr})(\mathrm{OR})_{2}\left(=\mathrm{CHR}^{\prime}\right),{ }^{2}$ but those catalyst systems are also highly sensitive to air and moisture, and are expensive. For practical use, isolation of alkylidene complexes is not necessary and introduction of appropriate supporting ligands is important. For example, $\mathrm{WOCl}_{4-n}(\mathrm{OAr})_{n} / \mathrm{R}_{3} \mathrm{SnH}$ is a good catalyst precursor for bulk ROMP of dicyclopentadiene (DCPD). ${ }^{3}$ We have been interested in the effect of chelating aryloxo ligands on the catalysis of tungsten compounds for ROMP. ${ }^{4-8}$ However, these complexes also contained other ancillary ligands such as oxo and substituted alkyne, which could effect on their ROMP catalyses rather than the chelating aryloxo ligands. In order to clarify the effect of the chelating ligands on ROMP catalysis, we systematically studied a series of tungsten(VI) chloride complexes having chelating aryloxo

${ }^{\dagger}$ To whom correspondence should be addressed. ligands without any other ligands. Here we chose a commercially available and inexpensive ligand, catechol, as a practical ancillary ligand for tungsten catalysts. The ROMP catalyses of the catecholatotungsten complexes were compared with those of the monodentate aryloxo complexes and $\mathrm{WCl}_{6}$.

\section{EXPERIMENTAL}

\section{General}

All the operations were performed under argon with standard Schlenk techniques. ${ }^{1} \mathrm{H}$ NMR spectra were recorded on a JEOL JNM-LA-400 spectrometer, and chemical shifts were calibrated with the residual protons $(\delta=7.26 \mathrm{ppm})$ in $\mathrm{CDCl}_{3}$. The average molecular weights and molecular weight distributions of the polymers were determined by Gel permeation chromatographic (GPC) analyses on a Tosoh model SC-8010 instrument equipped with a RI (Tosoh RI-8012) detector connected to the TSK gel columns G1000, G2500, G4000, and G7000 in THF at $40^{\circ} \mathrm{C}$. Standard polystyrenes were used for calibration. Toluene was purified by distillation from sodiumbenzophenone. Tungsten complexes $\mathrm{WCl}_{6-2 n}(\mathrm{cat})_{n}(\mathbf{1}$ : $n=1,2: n=2$, cat = catecholato $), \mathrm{WCl}_{2}\left({ }^{\mathrm{t}} \mathrm{Bucat}\right){ }_{2}(\mathbf{3}$, ${ }^{\mathrm{t}}$ Bucat $=3,5$-di-tert-butylcatecholato), $\mathrm{WCl}_{6-n}(\mathrm{dmp})_{n}$ (4: $n=1,5: n=2,3: n=3, \mathrm{dmp}=2,6$-dimethylphenoxo), were prepared according to literatures. $^{9-12}$ Norbornene (bicyclo[2.2.1] hept-2-ene) purchased from Aldrich Chemical Company, Inc. was refluxed over sodium and distilled prior to use. DCPD was supplied by Zeon Corporation Co., Ltd., and purified similarly. Triethylaluminum and modified 
methylaluminoxane (MMAO, type $3 \mathrm{~A}$ in toluene) ${ }^{13}$ were supplied by Tosoh-Finechem co., and used without further purification.

\section{Polymerization of Norbornene with Tungsten Complex/ $\mathrm{AlEt}_{3}$}

Into a schlenk tube were placed the catalyst $(0.02 \mathrm{mmol})$ and toluene $(1.0 \mathrm{~mL})$ to form a homogeneous solution. After the solution was cooled to $-78^{\circ} \mathrm{C}, \mathrm{AlEt}_{3}(40 \mathrm{mM}, 0.5 \mathrm{~mL})$ was added to the reactor, and the mixture was stirred for $30 \mathrm{~min}$ for activation. After the reaction mixture was warmed to room temperature, polymerization was started by introducing a norbornene solution $(4 \mathrm{M}, 0.5 \mathrm{~mL})$. The polymerization mixture was quenched with a large amount of methanol containing a small amount of hydrochloric acid. The precipitates were collected by centrifugation and dried in vacuo. NMR and GPC measurements of the polymers were performed.

\section{Polymerization of Norbornene with Tungsten Com- plex/MMAO}

Into a schlenk tube were placed the catalyst $(0.02 \mathrm{mmol})$ and toluene $(0.41 \mathrm{~mL})$ to form a homogeneous solution. After the solution was cooled to $-78^{\circ} \mathrm{C}$, MMAO $(5.7 \mathrm{wt} \%, 1.09 \mathrm{~mL})$ was added to the reactor, and the mixture was stirred for $30 \mathrm{~min}$ for activation. After the reaction mixture was warmed to room temperature, polymerization was started by introducing a norbornene solution $(4 \mathrm{M}, 0.5 \mathrm{~mL})$. The polymerization mixture was quenched with a large amount of methanol containing a small amount of hydrochloric acid. The precipitates were collected by centrifugation and dried in vacuo. NMR and GPC measurements of the polymers were performed.

\section{RESULTS AND DISCUSSION}

Chatecholatotungsten(VI) complexes, $\mathrm{WCl}_{6-2 n}(\mathrm{cat})_{n}$ (1: $n=1,2: n=2$, cat = catecholato $)$ and $\mathrm{WCl}_{2}-$ $\left({ }^{\mathrm{t}} \text { Bucat }\right)_{2} \quad\left(3,{ }^{\mathrm{t}}\right.$ Bucat $=3,5$-di-tert-butylcatecholato $)$, were prepared according to literatures (Scheme 1)., 10 For comparison, monodentate aryloxotungsten complexes, $\mathrm{WCl}_{6-n}(\mathrm{dmp})_{n}(\mathbf{4}: n=1, \mathbf{5}: n=2, \mathbf{6}: n=3$, $\mathrm{dmp}=2,6$-dimethylphenoxo), were also prepared (Scheme 1). ${ }^{11,12}$

\section{Polymerization of Norbornene with Tungsten Com- plexes/AlEt 3}

Catecholato complexes 1-3 were inactive for the ROMP of norbornene in the absence of cocatalyst, while one equivalent of $\mathrm{AlEt}_{3}$ effectively activated the catecholato complexes. Table I summarizes the poly-
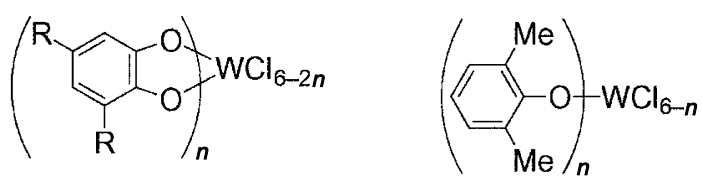

$$
\begin{aligned}
& \text { 1: } n=1, \mathrm{R}=\mathrm{H} \\
& \text { 2: } n=2, \mathrm{R}=\mathrm{H} \\
& \text { 3: } n=2, \mathrm{R}={ }^{\mathrm{t}} \mathrm{Bu}
\end{aligned}
$$$$
\begin{aligned}
& \text { 4: } n=1 \\
& \text { 5: } n_{1}=2 \\
& \text { 6: } n_{1}=3
\end{aligned}
$$

Scheme 1.

\begin{tabular}{|c|c|c|c|c|c|}
\hline Complex & $\begin{array}{c}\text { Temp. } \\
\left({ }^{\circ} \mathrm{C}\right)\end{array}$ & $\begin{array}{c}\text { Yield } \\
(\%)\end{array}$ & $\begin{array}{c}M_{\mathrm{n}}{ }^{\mathrm{a}} \\
\left(/ 10^{5}\right)\end{array}$ & $M_{\mathrm{w}} / M_{\mathrm{n}}^{\mathrm{a}}$ & $\begin{array}{c}\text { cis-cont. }^{\mathrm{b}} \\
(\%)\end{array}$ \\
\hline \multirow[t]{4}{*}{ (cat) $\mathrm{WCl}_{4}(\mathbf{1})$} & -78 & no polym. & - & - & - \\
\hline & 0 & 74 & 17.75 & 1.99 & 65 \\
\hline & 25 & 69 & 13.70 & 1.68 & 42 \\
\hline & 60 & 74 & 8.47 & 1.83 & 51 \\
\hline \multirow[t]{4}{*}{ (cat) $)_{2} \mathrm{WCl}_{2}(\mathbf{2})$} & -78 & trace & - & - & - \\
\hline & 0 & 98 & 3.55 & 1.31 & 73 \\
\hline & 25 & 85 & 2.94 & 1.40 & 69 \\
\hline & 60 & 98 & 4.71 & 1.89 & 53 \\
\hline \multirow[t]{4}{*}{$\left({ }^{t} \text { Bucat }\right)_{2} \mathrm{WCl}_{2}(\mathbf{3})$} & -78 & no polym. & - & - & - \\
\hline & 0 & 48 & 8.45 & 1.88 & 80 \\
\hline & 25 & 49 & 6.88 & 1.89 & 86 \\
\hline & 60 & 50 & 3.43 & 1.34 & 63 \\
\hline
\end{tabular}

Table I. ROMP of norbornene by tungsten complexes activated with $\mathrm{AlEt}_{3}$

Conditions; $[\mathrm{W}]=10 \mathrm{mM},[\mathrm{NBE}]_{\mathrm{o}}=1 \mathrm{M},\left[\mathrm{AlEt}_{3}\right]=10 \mathrm{mM}$,

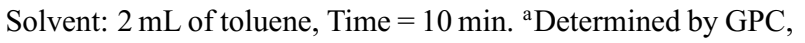
${ }^{b}$ Determined by ${ }^{1} \mathrm{H}$ NMR.

Table II. ROMP of norbornene by tungsten complexes activated with $\mathrm{AlEt}_{3}$

\begin{tabular}{lrcccc}
\hline \multicolumn{1}{c}{ Complex } & $\begin{array}{r}\text { Temp. } \\
\left({ }^{\circ} \mathrm{C}\right)\end{array}$ & $\begin{array}{c}\text { Yield } \\
(\%)\end{array}$ & $\begin{array}{c}M_{\mathrm{n}}{ }^{\mathrm{a}} \\
\left(/ 10^{5}\right)\end{array}$ & $M_{\mathrm{w}} / M_{\mathrm{n}}{ }^{\mathrm{a}}$ cis-cont. $^{\mathrm{b}}$ \\
$(\%)$
\end{tabular}

Conditions; $[\mathrm{W}]=10 \mathrm{mM},[\mathrm{NBE}]_{\mathrm{o}}=1 \mathrm{M}$, Solvent : $2 \mathrm{~mL}$ of toluene, Time $=10 \mathrm{~min} . \quad(\mathrm{dmp})=2,6$-dimethylphenoxy. ${ }^{\mathrm{a}}$ Determined by GPC, ${ }^{\mathrm{b}}$ Determined by ${ }^{1} \mathrm{H}$ NMR.

merization of norbornene by catecholato complexes 13 in the presence of $\mathrm{AlEt}_{3}$. Table II shows the polymerization by monodentate dmp complexes 4-6 and $\mathrm{WCl}_{6}$ for comparison. When $\mathrm{AlEt}_{3}$ was used as a co- 
catalyst, catecholato complexes $\mathbf{1}-\mathbf{3}$ showed high activities. Especially, the bis(catecholato) complex 2 was remarkably more active than any other catecholato, dmp complexes, and $\mathrm{WCl}_{6}$. When the monomer solution in toluene was added to the mixture of $2 / \mathrm{AlEt}_{3}$ in toluene, gelation of the reaction mixture immediately occurred. The molecular weights of the polymers obtained with 2/AlEt 3 were rather lower than those obtained with 1 and $3 / \mathrm{AlEt}_{3}$, indicating higher initiator efficiency of 2/AlEt 3 than complexes 1 and 3 systems. The polymers obtained with bis(catecholato) complexes $\mathbf{2}$ and $\mathbf{3}$ had significantly narrower molecular weight distributions than those obtained with $\mathrm{WCl}_{6}$. Chelating coordination of two catecholato ligands should be appropriate both for enhancement of the catalytic activity and for suppression of side reactions such as secondary metathesis. ${ }^{14}$ The mono(catecholato) complex 1 gave polymers with broader molecular weight distributions compared with the corresponding bis(catecholato) complex 2. Coordination of only one catecholato ligand in 1 might be insufficient for effective stabilization of the active species. The bulky bis(catecholato) complex 3 yielded polymers with relatively high cis-content, while the corresponding unsubstituted bis(catecholato) complex 2 did not show cis/trans selectivity. The bulky substituents of $\mathbf{3}$ were essential for control of the microstructure of the polymers, although the activity of $\mathbf{3}$ was lower than that of $\mathbf{2}$. Thus, chelating catecholato ligands showed better activitiy and control of ROMP than the monodentate dmp complexes (Table II).

Figure 1 shows GPC profiles of the polymers obtained with catecholato complexes $\mathbf{1}-\mathbf{3}$ and $\mathrm{WCl}_{6}$ based systems. The polymers obtained with complex $\mathbf{1}$ and $\mathrm{WCl}_{6}$ had shoulder peaks, indicating existence of two or more active species. In contrast, the bis(catecholato) complexes $\mathbf{2}$ and $\mathbf{3}$ based systems could be regarded as single site catalysts. The $\mathrm{AlEt}_{3}$ cocatalyst should alkylate the tungsten complexes followed by $\alpha$-H elimination to generate alkylidene species as catalytically active species. We are assuming that the alkylidene species from bis(catecholato) complexes $\mathbf{2}$ and $\mathbf{3}$ could be formulated as $\mathrm{WL}_{2}\left(=\mathrm{CHCH}_{3}\right)\left(\mathrm{L}=\right.$ cat or ${ }^{t}$ Bucat $)$, while the mono(catecholato)complex $1 / \mathrm{AlEt}_{3}$ could generate $\mathrm{WX}_{2}$ (cat) $\left(=\mathrm{CHCH}_{3}\right)(\mathrm{X}=\mathrm{Cl}$ and/or Et $)$ and $\mathrm{WCl}_{6} / \mathrm{AlEt}_{3}$ system could give a more complicated mixture. This multiplicity of active species in $\mathbf{1}$ and $\mathrm{WCl}_{6}$ systems should be the reason for the shoulder peaks of their GPC profiles.

The complexes 4-6 having monodentate dmp ligands also afforded polymers with narrower molecular weight distributions in comparison with $\mathrm{WCl}_{6}$ (Table II), but they were less active than bis(catecholato) complex 2. Among the three dmp complexes 4-6, bis(dmp) com-

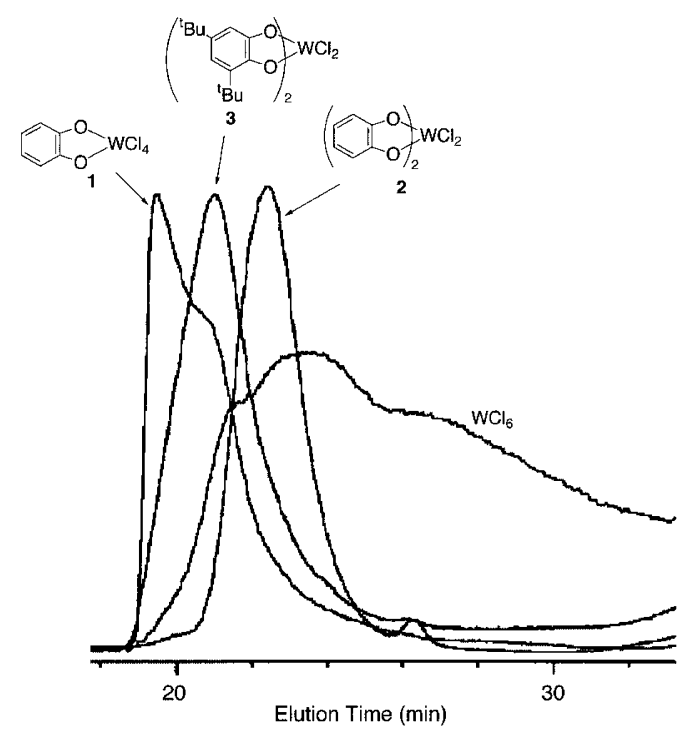

Figure 1. GPC profiles of poly(norbornene)s obtained with tungsten catalysts/ $\mathrm{AIEt}_{3}$ at $0{ }^{\circ} \mathrm{C}$.

plex 5 was most active. With increasing dmp ligands on tungsten, the molecular weights of the resulting polymers decreased, suggesting that the initiation became faster and propagation slower with increasing the dmp ligands. This could be attributed to the steric effect of the dmp ligands, which also enhanced cis selectivity with increasing the dmp ligands. It is noteworthy that only the monoaryloxo complex $\mathbf{4}$ gave polynorbornene at $60^{\circ} \mathrm{C}$ without any cocatalyst although the polymer yield was rather low $(22 \%$ yield under similar conditions in Table II except for the polymerization time of $3 \mathrm{~h})$.

\section{Polymerization of Norbornene with Tungsten Com- plexes/MMAO}

We have recently found that $\{$ bis(imino) pyridine $\} \mathrm{MoCl}_{3}$ (7)/MMAO system promoted ROMP of norbornene to give polymers with high cis-contents, while $7 / \mathrm{AlEt}_{3}$ resulted in low cis selectivity. ${ }^{15}$ This encouraged us to use MMAO as a cocatalyst for the complexes 1-6 (Tables III and IV). The polymerization of NBE by MMAO-activated systems also proceeded in ROMP fashion and vinyl addition polymerization of norbornene did not occur. The catecholato complexes 1-3/MMAO systems were active for the ROMP of NBE at $-78^{\circ} \mathrm{C}$. At this temperature, the bis(catecholato) complex 2/MMAO was particularly active, although the molecular weight distribution of the polymer was rather broad in comparison with those obtained with $2 / \mathrm{AlEt}_{3}$. On the other hand, the bulky complex $\mathbf{3}$ became most active among these complexes at $0-60^{\circ} \mathrm{C}$ to give polymers with relatively narrow molecular weight distributions. This might partly result from the thermal stabilities of 
Table III. ROMP of norbornene by tungsten complexes activated with MMAO

\begin{tabular}{|c|c|c|c|c|c|}
\hline Complex & $\begin{array}{l}\text { Temp. } \\
\left({ }^{\circ} \mathrm{C}\right)\end{array}$ & $\begin{array}{l}\text { Yield } \\
(\%)\end{array}$ & $\begin{array}{c}M_{\mathrm{n}}^{\mathrm{a}} \\
\left(/ 10^{5}\right)\end{array}$ & $M_{\mathrm{w}} / M_{\mathrm{n}}^{\mathrm{a}}$ & $\begin{array}{c}\text { cis-cont. } \\
(\%)\end{array}$ \\
\hline \multirow[t]{4}{*}{ (cat) $\mathrm{WCl}_{4}(\mathbf{1})$} & -78 & 40 & 2.44 & 1.82 & 53 \\
\hline & 0 & 84 & 2.85 & 1.63 & 43 \\
\hline & 25 & 71 & 3.83 & 1.46 & 44 \\
\hline & 60 & 20 & 3.62 & 1.46 & 45 \\
\hline \multirow[t]{4}{*}{$(\mathrm{cat})_{2} \mathrm{WCl}_{2}(\mathbf{2})$} & -78 & 86 & 2.02 & 1.74 & 45 \\
\hline & 0 & 65 & 1.57 & 1.27 & 44 \\
\hline & 25 & 63 & 2.26 & 1.30 & 52 \\
\hline & 60 & 74 & 1.79 & 1.31 & 36 \\
\hline \multirow[t]{4}{*}{$\left({ }^{\mathrm{t}} \text { Bucat }\right)_{2} \mathrm{WCl}_{2}(\mathbf{3})$} & -78 & 10 & 2.78 & 1.26 & 54 \\
\hline & 0 & $>99$ & 1.85 & 1.68 & 52 \\
\hline & 25 & 91 & 1.49 & 1.36 & 43 \\
\hline & 60 & $>99$ & 2.13 & 1.30 & 40 \\
\hline
\end{tabular}

Conditions; $[\mathrm{W}]=10 \mathrm{mM},[\mathrm{NBE}]_{\mathrm{o}}=1 \mathrm{M},[\mathrm{MMAO}]=1 \mathrm{M}$, Solvent : $2 \mathrm{~mL}$ of toluene, Time $=10 \mathrm{~min}$. ${ }^{\text {a Determined by }}$ GPC. ${ }^{b}$ Determined by ${ }^{1} \mathrm{H}$ NMR.

Table IV. ROMP of norbornene by tungsten complexes

\begin{tabular}{crcccc}
\multicolumn{5}{c}{ activated with MMAO } \\
\hline Complex & $\begin{array}{c}\text { Temp. } \\
\left({ }^{\circ} \mathrm{C}\right)\end{array}$ & $\begin{array}{c}\text { Yield } \\
(\%)\end{array}$ & $\begin{array}{c}M_{\mathrm{n}}{ }^{\mathrm{a}} \\
\left(/ 10^{5}\right)\end{array}$ & $M_{\mathrm{w}} / M_{\mathrm{n}}{ }^{\mathrm{a}}$ & $\begin{array}{c}\text { cis-cont. }^{\mathrm{b}} \\
(\%)\end{array}$ \\
\hline (dmp) $\mathrm{WCl}_{5}(\mathbf{4})$ & -78 & 33 & 2.29 & 1.44 & 41 \\
& 0 & 28 & 2.04 & 1.56 & 51 \\
& 25 & 66 & 2.31 & 1.54 & 31 \\
& 60 & 37 & 2.18 & 1.49 & 32 \\
\hline$(\mathrm{dmp})_{2} \mathrm{WCl}_{4}(\mathbf{5})$ & -78 & 11 & 2.02 & 1.50 & 63 \\
& 0 & 68 & 2.78 & 1.49 & 36 \\
& 25 & 79 & 2.71 & 1.49 & 30 \\
$-(\mathrm{dmp})_{3} \mathrm{WCl}_{3}(\mathbf{6})$ & -78 & 69 & 2.83 & 1.54 & 27 \\
& 0 & 26 & 2.33 & 1.55 & 57 \\
& 25 & 89 & 4.13 & 1.34 & 46 \\
60 & 67 & 4.62 & 1.33 & 28 \\
\hline
\end{tabular}

Conditions; $[\mathrm{W}]=10 \mathrm{mM},[\mathrm{NBE}]_{\mathrm{o}}=1 \mathrm{M},[\mathrm{MMAO}]=1 \mathrm{M}$, Solvent : $2 \mathrm{~mL}$ of toluene, Time $=10 \mathrm{~min}$. ${ }^{\text {a Determined by }}$ GPC. ${ }^{b}$ Determined by ${ }^{1} \mathrm{H}$ NMR.

the active species in these systems. The cis-contents of the polymers obtained with catecholatotungsten complex/MMAO systems were 36-54\%, lower than those obtained with the AlEt 3 -activated system. The structure of the active species in the MMAO systems should differ from that in the $\mathrm{AlEt}_{3}$ systems. In the polymerization by the monodentate phenolato complexes 4-6, the mono(dmp) complex 4/MMAO showed rather low activities, while activities of the bisand tris(dmp) complexes $\mathbf{5}$ and $\mathbf{6}$ were similar to those of bis(catecholato) complex 2.

\section{Polymerization of Dicyclopentadiene with Tungsten Complexes/AlEt 3}

The catecholato complexes/AlEt 3 systems were applied to the polymerization of dicyclopentadiene (DCPD) (Table V). The catecholato complexes 1-3
Table V. ROMP of dicyclopentadiene by tungsten complexes activated with $\mathrm{AlEt}_{3}$

\begin{tabular}{|c|c|c|c|c|c|c|}
\hline Complex & \multicolumn{2}{|c|}{ Temp. Time } & $\begin{array}{c}\text { Yield } \\
(\%)\end{array}$ & $\begin{array}{c}M_{\mathrm{n}}{ }^{\mathrm{a}} \\
\left(/ 10^{5}\right)\end{array}$ & $M_{\mathrm{w}} / M_{\mathrm{n}}^{\mathrm{a}}$ & $\begin{array}{c}\text { cis-cont. }^{\mathrm{b}} \\
(\%)\end{array}$ \\
\hline \multirow[t]{4}{*}{ (cat) $\mathrm{WCl}_{4}(\mathbf{1})$} & -78 & 24 & no polym. & - & - & - \\
\hline & 0 & 6 & 53 & 1.57 & 1.38 & 90 \\
\hline & 25 & 6 & 65 & 1.38 & 2.04 & 90 \\
\hline & 60 & 6 & 56 & 1.61 & 2.25 & 81 \\
\hline \multirow[t]{4}{*}{ (cat) $)_{2} \mathrm{WCl}_{2}(2)$} & -78 & 24 & no polym. & - & - & - \\
\hline & 0 & 24 & 3 & 6.49 & 1.67 & 93 \\
\hline & 25 & 24 & 29 & 3.76 & 1.56 & 88 \\
\hline & 60 & 24 & 56 & 1.23 & 2.52 & 93 \\
\hline \multirow[t]{4}{*}{$\left({ }^{\mathrm{t}} \mathrm{Bucat}\right)_{2} \mathrm{WCl}_{2}(\mathbf{3}$} & -78 & 24 & no polym. & - & - & - \\
\hline & 0 & 24 & 27 & 6.60 & 1.96 & 96 \\
\hline & 25 & 24 & 31 & 3.83 & 2.10 & 90 \\
\hline & 60 & 24 & 52 & 1.29 & 2.16 & 88 \\
\hline
\end{tabular}

Conditions; $[\mathrm{W}]=10 \mathrm{mM},[\mathrm{NBE}]_{\mathrm{o}}=1 \mathrm{M},[\mathrm{MMAO}]=1 \mathrm{M}$, Solvent : $2 \mathrm{~mL}$ of toluene, Time $=10 \mathrm{~min}$. ${ }^{\text {a Determined by }}$ GPC. ${ }^{b}$ Determined by ${ }^{1} \mathrm{H}$ NMR.

successfully polymerized DCPD to give soluble polymers. For the polymerization of this bulky monomer, less bulky monocatecholato complex 1 exhibited significantly higher activity than the bis(catecholato) complexes $\mathbf{2}$ and $\mathbf{3}$. Thus, the catalytic behavior of the catecholato complexes could be tuned by the number of the catecholato ligands depending on the monomer. The poly(DCPD)s obtained with these catalyst systems had high cis-contents in comparison with the poly(NBE)s.

\section{CONCLUSION}

Introduction of two catecholato ligands into the tungsten metal center is quite effective not only on the control of molecular weight distribution but also on the enhancement of the catalytic activity for ROMP of norbornene. Bis(catecholato) complex gave polymers with narrower molecular weight distributions than those observed in monodentate 2,6-dimethylphenoxo complexes. Chelating coordination and conjugated structure of catecholato ligands could adequately affect the reactivity of the active species. The use of bulky catecholato ligand ${ }^{t}$ Bucat resulted in higher cis-selectivity in comparison with unsubstituted catecholato ligand, although its activity was lower than the unsubstituted bis(catecholato) complex. In the case of polymerization of DCPD, mono(catecholato)complex was particularly active to give soluble poly(DCPD) with high ciscontent. Thus, catecholato ligands were found to be quite effective to improve ROMP catalysis of tungsten complexes as practical catalyst precursors.

Acknowledgment. This research was carried out partially financed by the NEDO International Joint Re- 
search Grant Program "Biodegradable Copolymers". We are indebted to a Grant-in-Aid for Scientific Research (Grant No.14044072 and 14350488) from the Ministry of Education, Culture, Sport, Science and Technology.

\section{REFERENCES AND NOTES}

1. K. J. Ivin and J. C. Mol, "Olefin Metathesis and Metathesis Polymerization”, Academic Press, London, 1997.

2. R. R. Schrock, Acc. Chem. Res., 23, 158 (1990).

3. A. Bell, J. Mol. Catal., 76, 165 (1992).

4. Y. Nakayama, N. Ikushima, and A. Nakamura, Chem. Lett., 861 (1997).

5. Y. Nakayama, H. Saito, N. Ueyama, and A. Nakamura, Organometallics, 18, 3149 (1999).

6. Y. Takashima, Y. Nakayama, H. Yasuda, A. Nakamura, and A. Harada, J. Organomet. Chem., 654, 74 (2002).
7. Y. Takashima, Y. Nakayama, H. Yasuda, and A. Harada, $J$. Organomet. Chem. 651, 114 (2002).

8. Y. Takashima, Y. Nakayama, H. Yasuda, A. Nakamura, and A. Harada, J. Organomet. Chem., 664, 234 (2002).

9. Y. Nakayama, H. Saito, and A. Nakamura, Chem. Lett., 691 (1996).

10. S. M. Beshouri and I. P. Rothwell, Inorg. Chem., 25, 1962 (1986).

11. H. Yasuda, Y. Nakayama, K. Takei, A. Nakamura, Y. Kai, and N. Kanehisa, J. Organomet. Chem., 473, 105 (1994).

12. F. Quignard, M. Leconte, J.-M. Basset, L.-Y. Hsu, J. J. Alexander, and S. G. Shore, Inorg. Chem., 26, 4272 (1987).

13. MMAO (type $3 \mathrm{~A}$ ) is a partially hydrolyzed product of a mixture of $\mathrm{AlMe}_{3}$ and $\mathrm{Al}^{\mathrm{i}} \mathrm{Bu}_{3}$, which can be formally described as a approximate formula, $\left[\mathrm{Me}_{0.7}{ }^{\mathrm{i}} \mathrm{Bu}_{0.3} \mathrm{AlO}\right]_{n}$.

14. J. Kress, J. Mol. Catal. A: Chem., 102, 7 (1995).

15. K. Hiya, Y. Nakayama, and H. Yasuda, Macromolecules, in press (2003). 